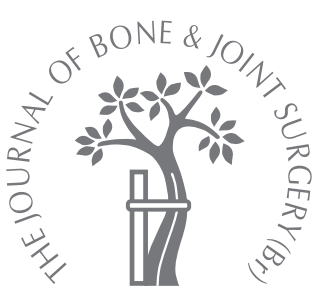

I. D. M. Smith, R. Elton, J. A. Ballantyne, I. J. Brenkel

From Queen Margaret Hospital, Dunfermline, Scotland

\title{
Pre-operative predictors of the length of hospital stay in total knee replacement
}

In Scotland, the number of primary total knee replacements performed annually has been increasing steadily. The price of the implant is fixed but the length of hospital stay is variable.

We prospectively investigated all patients who underwent primary unilateral total knee replacement in the Scottish region of Fife, between December 1994 and February 2007 and assessed their recorded pre-operative details. The data were analysed using univariate and multiple linear regression statistical analysis.

Data on the length of stay were available from a total of 2106 unilateral total knee replacements. The median length of hospital stay was eight days. The significant preoperative risk factors for an increased length of stay were the year of admission, details of the consultant looking after the patient, the stair score, the walking-aid score and age.

Awareness of the pre-operative factors which increase the length of hospital stay may provide the opportunity to influence them favourably and to reduce the time in hospital and the associated costs of unilateral total knee replacement.

In the United Kingdom more than 30000 total knee replacements (TKRs) are carried out annually. ${ }^{1}$ With an increase in the prevalence of arthritis and obesity, and with an ageing population, the demand for TKR is expected to increase. In Scotland, the number of primary TKRs carried out has been steadily increasing since 1992. ${ }^{2}$ Data from the Scottish Arthroplasty Project have predicted that the number performed annually will soon outstrip that of total hip replacements. ${ }^{2}$ This has already occurred in Australia during the period 2002 to 2003 . $^{2}$

It is estimated that TKRs now cost the National Health Service (NHS) more each year than hip replacements. ${ }^{1}$ Each implant costs approximately $£ 2500$. Additional costs are incurred through the stay in hospital and the post-operative rehabilitation of the patient. It is predicted that TKR will cost the NHS almost $£ 1$ billion per year by 2010 if it continues to increase at the present rate. ${ }^{1}$

The price of the implant is fixed, but the length of hospital stay and associated hospital costs are variable. An understanding of the factors affecting hospital stay is therefore important, both from the point of view of the patient and the health-care economy.

Our aim was to determine which preoperative factors influenced the length of hospital stay for TKR based on information from a regional arthroplasty database.

\section{Patients and Methods}

Between December 1994 and February 2007 all patients undergoing primary unilateral TKR in the region of Fife, Scotland, an area with a population of approximately 360000 , were entered prospectively into a regional arthroplasty database.

All the patients were seen at a preassessment clinic by a dedicated audit nurse and their pre-operative haemoglobin was checked. They were admitted either on the day of or the day before surgery. The data collected included age, gender, body mass index (BMI) and all components of the American Knee Society score (AKSS). ${ }^{3}$ These are summarised in Tables I to III.

The AKSS consists of two parts. The first is the knee score which considers pain, stability and range of movement with deductions for flexion contracture, extension lag and malalignment. A maximum of 100 points is given for a well-aligned knee with no pain, $125^{\circ}$ of movement and negligible anteroposterior or mediolateral instability.

The second part is the function score, which considers walking distance and stair-climbing with deductions for the use of a walking aid. The maximum function score is 100 , scored by an individual who is able to walk an unlimited distance and can ascend and descend stairs normally. However, we assessed individual compo- 


\begin{tabular}{|c|c|c|c|}
\hline Factor & Number & Mean LOHS (days) & p-value \\
\hline \multicolumn{4}{|l|}{ Age (yrs) } \\
\hline$<60$ & 294 & 7.8 & $<0.001$ \\
\hline 60 to 69 & 730 & 8.8 & $(<0.001)$ \\
\hline 70 to 80 & 843 & 9.7 & \\
\hline$>80$ & 229 & 11.8 & \\
\hline \multicolumn{4}{|l|}{ Gender } \\
\hline Male & 971 & 9.1 & $<0.001$ \\
\hline Female & 1129 & 9.5 & $(0.17)$ \\
\hline \multicolumn{4}{|l|}{ Body mass index } \\
\hline$<25$ & 332 & 9.7 & 0.31 \\
\hline 25 to 29 & 751 & 9.1 & * \\
\hline 30 to 35 & 592 & 9.0 & \\
\hline$>35$ & 290 & 9.3 & \\
\hline \multicolumn{4}{|l|}{ Year of admission } \\
\hline 1995 to 1997 & 405 & 11.8 & $<0.001$ \\
\hline 1998 to 2000 & 367 & 9.5 & $(0.001)$ \\
\hline 2001 to 2003 & 491 & 8.8 & \\
\hline 2004 to 2007 & 839 & 8.5 & \\
\hline \multicolumn{4}{|l|}{ Day of operation } \\
\hline Monday & 415 & 9.9 & $<0.001$ \\
\hline Tuesday & 204 & 9.1 & $(0.24)$ \\
\hline Wednesday & 565 & 8.6 & \\
\hline Thursday & 600 & 9.6 & \\
\hline Friday & 274 & 9.5 & \\
\hline
\end{tabular}

nents of the function score separately. The scores for walking distance, the use of walking aids and stair-climbing are given in Table IV.

Surgical data included the diagnosis, details and grade of the surgeon performing the operation, the year and day of the operation, and the date of discharge.

All surgery was performed under the supervision of one of 11 consultant surgeons. An all-cemented TKR was carried out using the Press Fit Condylar (PFC; DePuy International Ltd, Leeds, United Kingdom) knee. In 1998 the geometry of the femoral component was modified and the implant was renamed the PFC Sigma knee. Patellar resurfacing was not performed routinely. In all patients intravenous antibiotics were given prophylactically before application of the tourniquet and in all routine cases low-molecular-weight heparin was given as prophylaxis for deep-vein thrombosis. From 2004 onwards Fondaparinux (GlaxoSmithKline PLC, Brentford, United Kingdom) was used in high risk cases.

The patients were followed up at 6,18 and 36 months and after five years in a dedicated clinic. Complications were recorded. The endpoint of the study was the date of discharge from hospital and no patient was lost to follow-up. Statistical analysis. The length of hospital stay varied from four to 70 days. Since this was highly skewed, statistical analysis was based on the logarithm of the length of hospital stay, which had an approximately symmetrical distribution. The relationship between each factor and the length of stay was analysed separately using a $t$-test or the Pearson correlation. Multiple linear regression analysis was used for testing the effect of each significant factor adjusted for the others. As a result of the logarithmic transformation, the coefficients from this model converted to percentage differences in length of stay between categories for each factor.

Statistical analysis was performed using SPSS version 14.0 (SPSS Inc., Chicago, Illinois). A p-value $\leq 0.05$ was considered to be significant.

\section{Results}

Data on the length of hospital stay were available from a total of 2105 primary unilateral TKRs. In the same period 190 bilateral TKRs were performed but these were not included in the study. The mean hospital stay was for 9.4 days (4 to 70 ). Tables I to III give the individual categories for each factor, the number in each category, the mean length of stay for each category and the univariate and multivariate p-values (multivariate $\mathrm{p}$-values are shown in parentheses).

Highly significant predictors of an increased stay when considered independently included age, gender, the day of 
Table II. The effect of surgical factors on the length of hospital stay (LOHS) after primary unilateral total knee replacement. The p-values are shown for each factor separately, and also in parentheses for each significant factor adjusted for all other significant factors

\begin{tabular}{|c|c|c|c|}
\hline Factor & Number & Mean LOHS (days) & p-value \\
\hline \multicolumn{4}{|l|}{ Diagnosis } \\
\hline Osteoarthritis & 1937 & 9.3 & 0.20 \\
\hline Other & 144 & 10.0 & * \\
\hline \multicolumn{4}{|c|}{ Pre-operative haemoglobin level (g/dl) } \\
\hline$<12$ & 262 & 10.3 & $<0.001$ \\
\hline 12 to 12.9 & 398 & 10.1 & $(0.84)$ \\
\hline 13 to 14 & 437 & 9.1 & $(0.17)$ \\
\hline$>14$ & 766 & 9.3 & \\
\hline \multicolumn{4}{|l|}{ Surgeon grade } \\
\hline Consultant & 1237 & 9.2 & 0.015 \\
\hline Specialist & 440 & 9.7 & $(0.80)$ \\
\hline Junior & 380 & 9.2 & \\
\hline \multicolumn{4}{|l|}{ Consultant code } \\
\hline 1 & 469 & 8.5 & $<0.001$ \\
\hline 2 & 277 & 9.5 & $(<0.001)$ \\
\hline $3^{\dagger}$ & 130 & 11.2 & \\
\hline $4^{\dagger}$ & 70 & 9.2 & \\
\hline $5^{\dagger}$ & 219 & 10.5 & \\
\hline 6 & 376 & 9.4 & \\
\hline 7 & 205 & 9.6 & \\
\hline $8^{\dagger}$ & 45 & 14.9 & \\
\hline 9 & 100 & 7.8 & \\
\hline 10 & 101 & 7.4 & \\
\hline 11 & 98 & 8.0 & \\
\hline
\end{tabular}

* not included in multiple regression as not significant on its own

† non-knee arthroplasty specialist

operation, the year of admission, the consultant orthopaedic surgeon, the grade of the surgeon, the walking score, the stair score, the walking-aid score, the AKSS and the preoperative level of haemoglobin (Tables I to III).

When these significant factors were adjusted for the effect of the other factors, using multiple linear regression analysis, the age, the year of admission, the walking-aid score, the stair score and details of the consultant looking after the patient remained significant.

The confidence limits for the effect sizes of the highly significant factors are given in Table $\mathrm{V}$ and are expressed as a percentage difference from either a reference category or a unit of quantitative measure. An increase in age of ten years was associated with an increased length of stay of between $10 \%$ and $13 \%$. The effect size of comparisons between different consultants was illustrated using two comparisons and were between $9 \%$ and $23 \%$ and $32 \%$ and $60 \%$, respectively in the two comparisons shown. The difference in the length of stay between consultants did not appear to be explained by any other factors.

\section{Discussion}

In our study, the length of stay was extremely variable and to our knowledge, this is the first attempt to address specif- ically pre-operative predictors of hospital stay in patients with primary unilateral TKR. Inevitably, higher costs are incurred in those patients who remain in hospital for longer periods of time. Awareness of those factors which result in an increased stay may allow better planning and a reduction in the length of stay after TKR.

For every advancing decade of age, the length of stay increased by $10 \%$ to $13 \%$ (Table I). This may have reflected the numerous co-morbidities and the lower level of fitness associated with increasing age. Moreover, elderly patients were at greater risk of peri-operative complications, such as sepsis, ${ }^{4}$ which were likely to extend their time in hospital.

Men had a significantly shorter stay in the univariate analysis (Table I). However, the gender difference did not remain significant when corrected for other variables. The year of admission was also significant (Table I). For each year that passed, there was a reduction of $1 \%$ to $2 \%$ at each time interval (Fig. 1). This downward trend has been noted in the Scottish Arthroplasty Project ${ }^{2}$ which has also shown a reduction on a year-to-year basis at national level. The current nationwide drive to reduce the length of stay in all patients is probably a contributory factor to the continuing reduction. ${ }^{5}$ Less invasive approaches to arthroplasty and advances in post-operative analgesia may also have an effect. 
Table III. The effect of the individual components of the American Knee score on the length of hospital stay (LOHS). The p-values are shown for each factor separately, and also in parentheses for each significant factor adjusted for all other significant factors

\begin{tabular}{|c|c|c|c|}
\hline Factor & Number & Mean LOHS (days) & p-value \\
\hline \multicolumn{4}{|l|}{ Knee score } \\
\hline$<20$ & 393 & 10.4 & $<0.001$ \\
\hline 20 to 34 & 641 & 9.4 & $(0.06)$ \\
\hline 35 to 45 & 529 & 9.2 & \\
\hline$>45$ & 522 & 8.8 & \\
\hline \multicolumn{4}{|c|}{ Walking score } \\
\hline 1 & 65 & 8.3 & $<0.001$ \\
\hline 2 & 149 & 8.7 & $(0.12)$ \\
\hline 3 & 724 & 9.1 & \\
\hline 4 & 1011 & 9.4 & \\
\hline 5 & 145 & 11.6 & \\
\hline 6 & 6 & 11.8 & \\
\hline \multicolumn{4}{|c|}{ Walking-aid score } \\
\hline 1 & 805 & 9.2 & $<0.001$ \\
\hline 2 & 766 & 9.5 & $(<0.001)$ \\
\hline 3 & 68 & 9.7 & \\
\hline 4 & 75 & 11.6 & \\
\hline 5 & 168 & 10.9 & \\
\hline \multicolumn{4}{|l|}{ Stair score } \\
\hline 1 & 64 & 8.0 & $<0.001$ \\
\hline 2 & 1076 & 8.8 & $(0.001)$ \\
\hline 3 & 897 & 9.9 & \\
\hline 4 & 9 & 11.7 & \\
\hline 5 & 54 & 12.9 & \\
\hline
\end{tabular}

Table IV. Scores designated for walking ability, walking-aids and stairs

\begin{tabular}{ll}
\hline Category & Score \\
\hline Walking ability & 1 \\
Unlimited & 2 \\
$>10$ blocks (1 hr) & 3 \\
5 to 10 blocks (10 to 30 mins) & 4 \\
$<5$ blocks (< 10 mins) & 5 \\
Housebound & 6 \\
Unable to walk & \\
& \\
Walking aids & 1 \\
No aid & 2 \\
One stick & 3 \\
Two sticks & 4 \\
Crutches/walker & 5 \\
Unable to walk & \\
Stairs & \\
Normal up and down & 1 \\
Normal up, and down with rail & 2 \\
Both up and down with rail & 3 \\
Up with rail, and unable to go down & 4 \\
Unable to manage stairs & 5 \\
\hline
\end{tabular}

Patients who underwent TKR performed by a consultant surgeon had a significantly reduced time in hospital in the univariate analysis (Table II). This almost certainly reflected
Table V. Confidence limits for effect sizes of highly significant factors adjusted for one another

\begin{tabular}{lll}
\hline Factor & Comparison & Confidence limits \\
\hline Age & Increase of ten years & +1 - to +13 \\
Year of admission & Increase of one year & -2 to -1 \\
Consultant & Code 3 vs code 1 & +9 to +23 \\
& Code 8 vs code 1 & +32 to +100 \\
Walking-aid score & Increase of one unit & +1 to +4 \\
Stairs score & Increase of one unit & +3 to +9 \\
\hline
\end{tabular}

the greater operative experience of a consultant in comparison with that of junior staff. However, the difference did not remain significant in the multiple linear regression analysis.

The variability in the length of stay between consultants within the same department (Table II; Fig. 2) was of interest. The three consultants with the longest period (3, 5 and 8 ) have now retired and did not specialise in knee arthroplasty. The consultants with a specialised interest in knee arthroplasty $(1,2,6,7,9$ and 10) were associated with significantly shorter lengths of stay than those who did not (3, 4, 5 and 8). Consultant 1 , a dedicated knee arthroplasty surgeon performed the greatest number of TKRs during the period of study and his patients had a mean length of stay of 8.5 days (Table V). When consultants 3 and 8 , non-knee specialists with the longest length of stay, were compared with consultant 1 , their patients had an increased time in hospital of between $9 \%$ and $23 \%$ and $32 \%$ and $60 \%$, respectively (Table V).

Although the day of the week on which surgery was performed was significant in the univariate analysis, it did not turn out to be so in the multiple linear regression analysis (Table I). It had been hitherto believed that those patients undergoing TKR at the beginning of the week would have the benefit of continuous rehabilitation for the remaining working days and would therefore have a shorter length of stay. However, this did not prove to be the case and may reflect on the efforts of the weekend medical and nursing staff to discharge patients. During the period of the study, our unit did not have orthopaedic physiotherapists working at the weekend.

The AKSS is a validated outcome measure for knee arthroplasty. ${ }^{3}$ It was a significant predictor of the length of stay in the univariate analysis but did not remain significant in the multiple linear regression analysis (Table III). However, the stair and walking-aid scores, two of the three components of the function score of the AKSS, were significant in the multiple linear regression analysis (Table III). For every increase in unit for the walking-aid score, the length of stay increased by $1 \%$ to $4 \%$ while it increased by $3 \%$ to $9 \%$ for every increasing unit in the stair score.

The significance of the walking-aid score may be due to the fact that those patients who required a walking aid pre-operatively became dependent on it both physically and psychologically. They had less confidence when it 


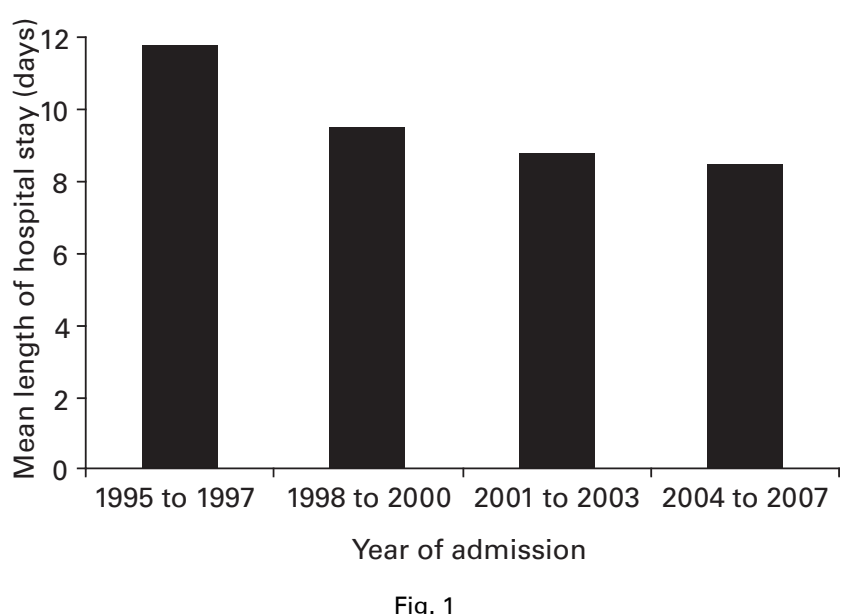

Bar chart showing the mean length of hospital stay in the period 1995 to 2007.

came to mobilising after surgery. Likewise, one of the last functional milestones required before discharge is being safe on the stairs. If a patient had difficulty with stairs pre-operatively then it was more likely that he or she would do so after surgery.

Surprisingly, the BMI was not a significant predictor of increased length of stay (Table I). A greater body-weight could be associated with more difficult surgery. ${ }^{6}$ Furthermore, such patients may be expected to have more problems mobilising after operation and to be more susceptible to post-operative complications. A study performed by Sadr Azodi et $\mathrm{al}^{7}$ investigated the impact of the BMI on the length of stay and the risk of post-operative complications among patients undergoing total hip replacement. They showed that a high BMI was significantly associated with an increased mean length of stay of between $4.7 \%$ and $7.0 \%$. The risk of systemic complications was increased by $58 \%$ in the obese. By contrast, a study of patients undergoing TKR by Amin et $\mathrm{al}^{6}$ showed that a BMI of greater than 30 did not increase the complications or the rate of re-operation.

The pre-operative level of haemoglobin was found to influence the length of stay in the univariate analysis (Table I). Patients with a pre-operative haemoglobin of less than $12 \mathrm{~g} / \mathrm{dl}$ had, on average, an increased length of stay of one day, compared with those with a pre-operative haemoglobin value greater than $13 \mathrm{~g} / \mathrm{dl}$. Patients with a lower pre-operative haemoglobin often have a lower postoperative value which requires blood transfusion. In a recent study, Guerin et $\mathrm{al}^{8}$ demonstrated that the pre-operative haemoglobin level of the patient was the only variable to predict independently the need for blood transfusion after primary hip or knee arthroplasty. Current guidelines suggest that pre-operative transfusion should only be undertaken under strict conditions. ${ }^{9,10}$

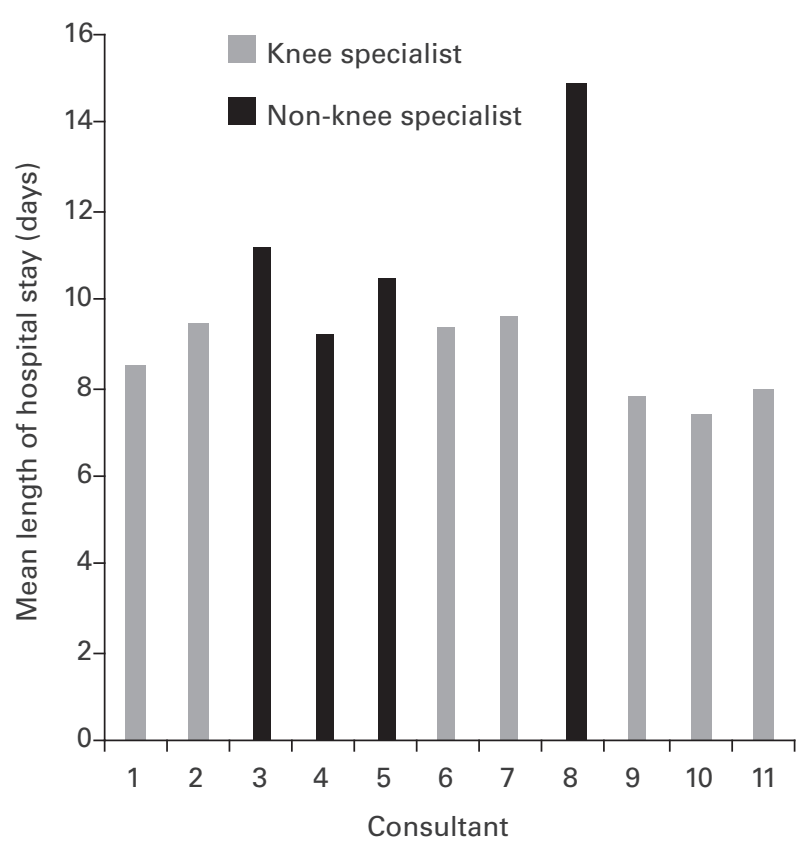

Fig. 2

Bar chart showing the mean for all the consultant orthopaedic surgeons involved in the study.

Our study has shown that a number of pre-operative factors influence the length of hospital stay. In some, the difference in the mean length of stay between categories of significant variables, i.e. gender, is less than 0.5 days. In our study, we preferred to use the mean as the appropriate summary statistic, in spite of the skewed nature of the distribution of length of stay, since this translated more directly into cost per patient. We accept that the cost of one half-day extra may not be significant in an individual patient, but would give rise to a significant cost over time.

Some of the pre-operative factors which influence the length of hospital stay such as the age of the patient, the use of a walking aid, difficulty with stairs, a haemoglobin level of less than $12 \mathrm{~g} / \mathrm{dl}$ and details regarding the surgeon, could be targeted to decrease it. Therefore, in our unit patients with a haemoglobin value of less than $12 \mathrm{~g} / \mathrm{dl}$ will be investigated and corrected if possible. We hope that this will decrease the transfusion rate and improve the length of stay. We will also target elderly patients pre- and postoperatively and only surgeons with an interest in knee arthroplasty will perform the surgery. We intend to re-audit the length of hospital stay in two to three years to determine the effects that these changes may have.

Although none of the authors has received or will receive benefits for personmal or professional use from a commercial party related directly or indirectly to the subject of this article, benefits have been received from DePuy to support the audit nurse. 


\section{References}

1. No authors listed. www.news.bbc.co.uk/1/health/3307887.stm (date last accessed 6 August 2008)

2. No authors listed. www.arthro.scot.nhs.uk/Reports/Scottish_Arthroplasty Project_Report_2007.pdf (date last accessed 6 August 2008).

3. Liow YL, Walker K, Wajid MA, Bedi G, Lennox CM. The reliability of the American Knee Society Score. Acta Orthop Scand 2000;71:603-8.

4. Parker MJ, Gurusamy K, Stoker M. Surgery in elderly patients. Current Orthopaedics 2004;18:333-44.

5. No authors listed. www.reducinglengthofstay.org.uk (date last accessed 6 August 2008)
6. Amin AK, Patton JT, Cook RE, Brenkel IJ. Does obesity influence the clinical outcome at five years following total knee replacement for osteoarthritis. J Bone Joint Surg [Br] 2006:88-B:335-40.

7. Sadr Azodi O, Bellocco R, Eriksson K, Adami J. The impact of tobacco use and body mass index on the length of stay in hospital and the risk of post-operative complications among patients undergoing total hip replacement. J Bone Joint Surg [Br]2006;88-B:1316-20.

8. Guerin S, Collins C, Kapoor H, McClean I. Collins D. Blood transfusion requirement prediction in patients undergoing primary total hip and knee arthroplasty. Transfusion Med 2007;17:37-43.

9. No authors listed. www.sign.ac.uk/pdf/sign54.pdf (date last accessed 6 August 2008)

10. No authors listed. www.asahq.org (date last accessed 6 August 2008). 